\title{
A New Application of the Reproducing Kernel Hilbert Space Method to Solve MHD Jeffery-Hamel Flows Problem in Nonparallel Walls
}

\author{
Mustafa Inc, ${ }^{1}$ Ali Akgül, ${ }^{2,3}$ and Adem Kılıçman ${ }^{4}$ \\ ${ }^{1}$ Department of Mathematics, Science Faculty, Fırat University, 23119 Elazı̆̆, Turkey \\ ${ }^{2}$ Department of Mathematics, Education Faculty, Dicle University, 21280 Diyarbakır, Turkey \\ ${ }^{3}$ Department of Mathematics and Statistics, Missouri University of Science and Technology, Rolla, MO 65409-0020, USA \\ ${ }^{4}$ Department of Mathematics and Institute for Mathematical Research, University Putra Malaysia, 43400 Serdang, Malaysia
}

Correspondence should be addressed to Adem Kılıçman; akilicman@putra.upm.edu.my

Received 9 February 2013; Accepted 28 February 2013

Academic Editor: Hassan Eltayeb

Copyright (C) 2013 Mustafa Inc et al. This is an open access article distributed under the Creative Commons Attribution License, which permits unrestricted use, distribution, and reproduction in any medium, provided the original work is properly cited.

\begin{abstract}
The present paper emphasizes Jeffery-Hamel flow: fluid flow between two rigid plane walls, where the angle between them is $2 \alpha$. A new method called the reproducing kernel Hilbert space method (RKHSM) is briefly introduced. The validity of the reproducing kernel method is set by comparing our results with HAM, DTM, and HPM and numerical results for different values of $H, \alpha$, and Re. The results show up that the proposed reproducing kernel method can achieve good results in predicting the solutions of such problems. Comparison between obtained results showed that RKHSM is more acceptable and accurate than other methods. This method is very useful and applicable for solving nonlinear problems.
\end{abstract}

\section{Introduction}

1.1. Problem Formulation. Consider a system of cylindrical polar coordinates $(r, h, z)$, where the steady two-dimensional flow of an incompressible conducting viscous fluid from a source or sink at channel walls lies in planes and intersects in $z$-axis. It is assumed that there are no changes with respect to $z$, that the motion is purely in radial direction and merely depends on $r$ and $\theta$, and that there is no magnetic field along $z$-axis. Then the governing equations are given as [1].

$$
\begin{gathered}
\frac{\rho \partial}{r \partial r}(r u(r, \theta))=0, \\
u(r, \theta) \frac{\partial u(r, \theta)}{\partial r}=v\left[\frac{\partial^{2} u(r, \theta)}{\partial r^{2}}+\frac{1}{r} \frac{\partial u(r, \theta)}{\partial r}\right. \\
\left.+\frac{1}{r^{2}} \frac{\partial^{2} u(r, \theta)}{\partial \theta^{2}}-\frac{u(r, \theta)}{r^{2}}\right] \\
-\frac{\sigma B_{0}^{2}}{\rho r^{2}} u(r, \theta)-\frac{1}{\rho} \frac{\partial P}{\partial r}
\end{gathered}
$$

$$
\frac{1}{\rho r} \frac{\partial P}{\partial \theta}-\frac{2 v}{r^{2}} \frac{\partial u(r, \theta)}{\partial \theta}=0
$$

where $B_{0}$ is the electromagnetic induction, $\sigma$ is the conductivity of the fluid, $u(r, \theta)$ is the velocity along radial direction, $P$ is the fluid pressure, $v$ is the coefficient of kinematic viscosity, and $\rho$ is the fluid density. From (1)

$$
f(\theta)=r u(r, \theta),
$$

using dimensionless parameters

$$
f(x)=\frac{f(\theta)}{f_{\max }}, x=\frac{\theta}{\alpha}
$$

where $\alpha$ is the semiangle between the two inclined walls as shown in Figure 1. Substituting (5) into (2) and (3) and eliminating $P$, we obtain an ordinary differential equation for the normalized function profile $F(x)[2]$ :

$$
F^{\prime \prime \prime}(x)+2 \alpha \operatorname{Re} F(x) F^{\prime}(x)+(4-H) \alpha^{2} F^{\prime}(x)=0,
$$


with boundary conditions

$$
F(0)=1, \quad F^{\prime}(0)=0, \quad F(1)=0 .
$$

The Reynolds number is

$$
\begin{aligned}
\operatorname{Re} & =\frac{f_{\max } \alpha}{v}=\frac{U_{\max } r \alpha}{v} \\
& =\left(\begin{array}{l}
\text { divergent channel: } \alpha>0, f_{\max }>0 \\
\text { convergent channel: } \alpha<0, f_{\max }<0
\end{array}\right) .
\end{aligned}
$$

The Hartmann number is

$$
H=\sqrt{\frac{\alpha B_{0}^{2}}{\rho v}} .
$$

Internal flow between two plates is one of the most applicable cases in mechanics, civil and environmental engineering. In simple cases, the one-dimensional flow through tube and parallel plates, which is known as CouettePoisseuille flow, has exact solution, but in general, like most of fluid mechanics equations, a set of nonlinear equations must be solved which make some problems for analytical solution. Many authors have shown interest in studying two-dimensional incompressible flow between two inclined plates. Jeffery [1] and Hamel et al. [2] were the first persons who discussed this problem, and so, it is known as Jeffery-Hamel problem. The incompressible viscous fluid flow through convergent and divergent channels is one of the most applicable cases in fluid mechanics, electrical, and biomechanical engineering. The MHD Jeffery-Hamel flows in nonparallel walls were investigated analytically for strongly nonlinear ordinary differential equations using homotopy analysis method (HAM). Results for velocity profiles in divergent and convergent channels were proffered for various values of Hartmann and Reynolds numbers in [3]. The mathematical investigations of this problem were underresearched by $[3,4]$. Jeffery-Hamel flows are of the NavierStokes equations in the particular case of two dimensional flow through a channel with inclined walls [3-13]. One of the most important examples of Jeffery-Hamel problems is this subjected to an applied magnetic field. The equations of magnetohydrodynamics have been solved exactly for the case of two-dimensional steady flow between nonparallel walls of a viscous, incompressible, electrically conducting fluid; this is a straightforward extension of the famous JeffreyHamel problem in ordinary hydrodynamics [9]. It has been indicated that for the Jeffrey-Hamel problem, the equations of magnetohydrodynamics can be curtailed to a set of three ordinary differential equations, two of which are linear and of first order [10]. In addition, these kinds of problems have been well studied in literature [3-13]. Most recent problems such as Jeffery-Hamel flow and other fluid mechanic problems are inherently nonlinear. Except a limited number of these problems, most of them do not have analytical solutions. So, these nonlinear equations should be solved utilizing other methods.

In this paper, the RKHSM [14-31] will be used to investigate MHD Jeffery-Hamel flows Problem. In recent years, a lot of attention has been devoted to the study of RKHSM to investigate various scientific models. The RKHSM which accurately computes the series solution is of great interest to applied sciences. The method provides the solution in a rapidly convergent series with components that can be elegantly computed.

Recently, a lot of research work has been devoted to the application of RKHSM to a wide class of stochastic and deterministic problems involving fractional differential equation, nonlinear oscillator with discontinuity, singular nonlinear two-point periodic boundary value problems, integral equations and nonlinear partial differential equations and so on [14-31]. The method is well suited to physical problems since it makes unnecessary restrictive methods.

The efficiency of the method was used by many authors to investigate several scientific applications. Cui and Lin [15] applied the RKHSM to handle the second-order boundary value problems. Wang et al. [24] investigated a class of singular boundary value problems by this method, and the obtained results were good. In [27], the method was used to solve nonlocal boundary value problems. Geng and Cui [18] investigated the approximate solution of the forced Duffing equation with integral boundary conditions by combining the homotopy perturbation method and the RKHSM. Recently, the method was appllied the fractional partial differential equations and multipoint boundary value problems [18-22]. For more details about RKHSM and the modified forms and its effectiveness, see [14-31] and the references therein. The paper is organized as follows. Section 2 is devoted to several reproducing kernel spaces. Solution representation in $W_{2}^{4}[0,1]$ and a linear operator are introduced in Section 3. Section 4 provides the main results; the exact and approximate solution of system (34) and an iterative method are developed for the kind of problems in the reproducing kernel space. We have proved that the approximate solution converges to the exact solution uniformly. Numerical results are given in Section 5. The last Section is the conclusions.

\section{Preliminaries}

2.1. Reproducing Kernel Spaces. In this section, we define some useful reproducing kernel spaces.

Definition 1 (reproducing kernel). Let $E$ be a nonempty abstract set. A function $K: E \times E \rightarrow C$ is a reproducing kernel of the Hilbert space $H$ if and only if

$$
\begin{gathered}
\forall t \in E, \quad K(\cdot, t) \in H, \\
\forall t \in E, \forall \varphi \in H, \quad(\varphi(\cdot), K(\cdot, t))=\varphi(t) .
\end{gathered}
$$

The last condition is called "the reproducing property"; the value of the function $\varphi$ at the point $t$ is reproduced by the inner product of $\varphi$ with $K(\cdot, t)$. 


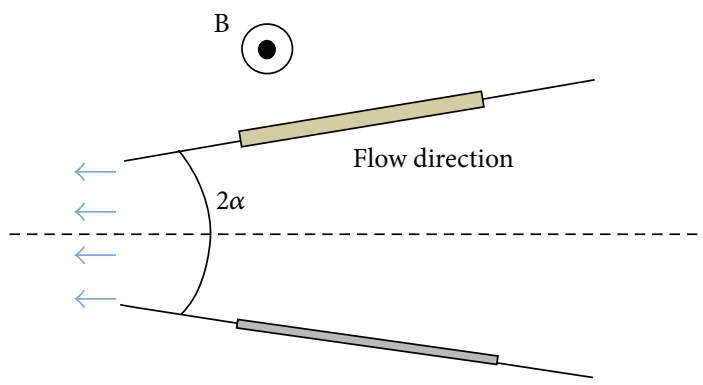

(a)

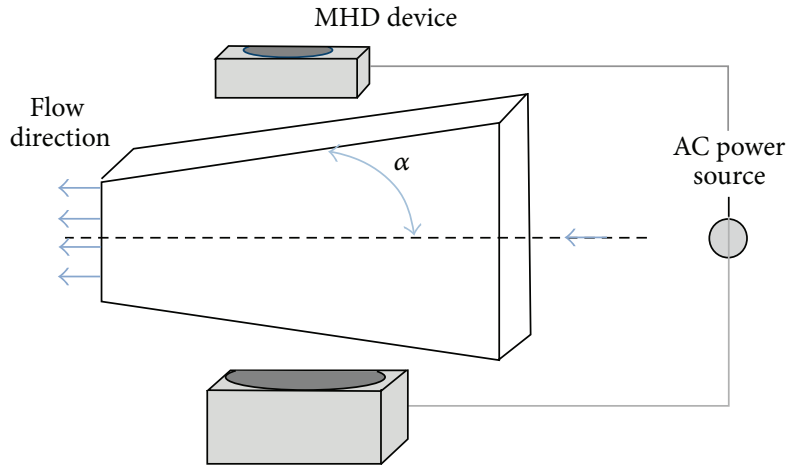

(b)

FIGURE 1: Geometry of the MHD Jeffery-Hamel flow in convergent cannel. (a) 2D view and (b) schematic setup of problem.

Definition 2. We define the space $W_{2}^{4}$ by

$$
W_{2}^{4}[0,1]=\left\{\begin{array}{l}
u \mid u, u^{\prime}, u^{\prime \prime}, u^{\prime \prime \prime}, \\
\quad \text { are absolutely continuous in }[0,1] \\
u^{(4)} \in L^{2}[0,1], x \in[0,1], \\
u(0)=0, u(1)=0, u^{\prime}(0)=0 .
\end{array}\right\} .
$$

The inner product and the norm in $W_{2}^{4}[0,1]$ are defined, respectively, by

$$
\begin{aligned}
&\langle u, g\rangle_{W_{2}^{4}}= \sum_{i=0}^{3} u^{(i)}(0) g^{(i)}(0) \\
&+\int_{0}^{1} u^{(4)}(x) g^{(4)}(x) d x, \quad u, g \in W_{2}^{4}[0,1], \\
&\|u\|_{W_{2}^{4}}=\sqrt{\langle u, u\rangle_{W_{2}^{4}}}, u \in W_{2}^{4}[0,1] .
\end{aligned}
$$

The space $W_{2}^{4}[0,1]$ is a reproducing kernel space; that is, for each fixed $y \in[0,1]$ and any $u(x) \in W_{2}^{4}[0,1]$, there exists a function $R_{y}(x)$ such that

$$
u(y)=\left\langle u, R_{y}\right\rangle_{W_{2}^{4}}
$$

Definition 3. We define the space $W_{2}^{2}$ by

$$
W_{2}^{2}[0,1]=\left\{\begin{array}{c}
u \mid u, u^{\prime} \text { are absolutely continuous in }[0,1] \\
u^{\prime \prime} \in L^{2}[0,1], x \in[0,1] .
\end{array}\right\}
$$

The inner product and the norm in $W_{2}^{2}[0,1]$ are defined, respectively, by

$$
\begin{array}{r}
\langle u, g\rangle_{W_{2}^{2}}=u(0) g(0)+u^{\prime}(0) g^{\prime}(0)+\int_{0}^{1} u^{\prime \prime}(x) g^{\prime \prime}(x) d x, \\
\left(u, g \in W_{2}^{2}[0,1]\right),
\end{array}
$$

$$
\|u\|_{W_{2}^{2}}=\sqrt{\langle u, u\rangle_{W_{2}^{2}}}, \quad u \in W_{2}^{2}[0,1] .
$$

The space $W_{2}^{2}[0,1]$ is a reproducing kernel space and its reproducing kernel function $T_{x}$ is given by

$$
T_{x}(y)= \begin{cases}1+x y+\frac{y}{2} x^{2}-\frac{1}{6} x^{3}, & x \leq y, \\ 1+x y+\frac{x}{2} y^{2}-\frac{1}{6} y^{3}, & x>y .\end{cases}
$$

Theorem 4. The space $W_{2}^{4}[0,1]$ is a complete reproducing kernel space; that is, for each fixed $y \in[0,1]$, there exists $u(x) \in W_{2}^{4}[0,1]$, such that

$$
u(y)=\left\langle u, R_{y}\right\rangle_{W_{2}^{4}}
$$

for any $u(x) \in W_{2}^{4}[0,1]$. The reproducing kernel $R_{y}$ can be denoted by

$$
R_{y}(x)= \begin{cases}\sum_{i=1}^{8} c_{i}(y) x^{i-1}, & x \leq y \\ \sum_{i=1}^{8} d_{i}(y) x^{i-1}, & x>y\end{cases}
$$

where

$$
c_{1}(y)=0,
$$

$c_{2}(y)=0$, 


$$
\begin{aligned}
& c_{3}(y)=\frac{21}{5680} y^{5}+\frac{1}{5680} y^{7}-\frac{7}{1136} y^{4}-\frac{7}{284} y^{3} \\
& +\frac{2}{71} y^{2}-\frac{7}{5680} y^{6} \\
& c_{4}(y)=\frac{7}{17040} y^{5}+\frac{1}{51120} y^{7}-\frac{7}{10224} y^{4}+\frac{16}{639} y^{3} \\
& -\frac{7}{284} y^{2}-\frac{7}{51120} y^{6} \\
& c_{5}(y)=\frac{7}{68160} y^{5}+\frac{1}{204480} y^{7}-\frac{7}{40896} y^{4}+\frac{4}{639} y^{3} \\
& -\frac{7}{1136} y^{2}-\frac{7}{204480} y^{6} \\
& c_{6}(y)=\frac{-7}{113600} y^{5}-\frac{1}{340800} y^{7}+\frac{7}{68160} y^{4}+\frac{7}{17040} y^{3} \\
& -\frac{1}{2130} y^{2}+\frac{7}{340800} y^{6} \\
& c_{7}(y)=\frac{7}{340800} y^{5}+\frac{1}{1022400} y^{7}-\frac{7}{204480} y^{4}-\frac{7}{51120} y^{3} \\
& -\frac{7}{5680} y^{2}-\frac{7}{1022400} y^{6}+\frac{1}{720} y \\
& c_{8}(y)=\frac{-1}{340800} y^{5}-\frac{1}{7156800} y^{7}+\frac{7}{204480} y^{4}+\frac{1}{51120} y^{3} \\
& +\frac{1}{5680} y^{2}+\frac{1}{10224000} y^{6}-\frac{1}{5040} \text {, } \\
& d_{1}(y)=\frac{-1}{5040} y^{7} \\
& d_{2}(y)=\frac{1}{720} y^{6}, \\
& d_{3}(y)=\frac{-1}{2130} y^{5}+\frac{1}{5680} y^{7}-\frac{7}{1136} y^{4}-\frac{7}{284} y^{3}+\frac{7}{21} y^{2} \\
& -\frac{7}{5680} y^{6} \\
& d_{4}(y)=\frac{4}{639} y^{4}+\frac{1}{17040} y^{5}+\frac{7}{40896} y^{7}+\frac{16}{639} y^{3} \\
& -\frac{7}{284} y^{2}-\frac{7}{51120} y^{6} \\
& d_{5}(y)=\frac{7}{68160} y^{5}+\frac{1}{24480} y^{7}-\frac{7}{40896} y^{4}-\frac{7}{10224} y^{3} \\
& -\frac{7}{1136} y^{2}-\frac{7}{204480} y^{6} \\
& d_{6}(y)=\frac{-7}{113600} y^{5}-\frac{1}{340800} y^{7}+\frac{7}{68160} y^{4}+\frac{7}{17040} y^{3} \\
& +\frac{21}{5680} y^{2}+\frac{7}{340800} y^{6} \\
& d_{7}(y)=\frac{7}{34080} y^{5}+\frac{1}{1022400} y^{7}-\frac{7}{204480} y^{4}-\frac{7}{51120} y^{3} \\
& -\frac{7}{5680} y^{2}-\frac{7}{1022400} y^{6} \text {, }
\end{aligned}
$$

$$
\begin{aligned}
d_{8}(y)= & \frac{-1}{340800} y^{5}-\frac{1}{7156800} y^{7}+\frac{1}{204480} y^{4}+\frac{1}{51120} y^{3} \\
& +\frac{1}{5680} y^{2}+\frac{7}{1022400} y^{6}
\end{aligned}
$$

Proof. By Definition 3, we have

$$
\begin{array}{r}
\left\langle u, R_{y}\right\rangle_{W_{2}^{4}}=\sum_{i=0}^{3} u^{(i)}(0) R_{y}^{(i)}(0)+\int_{0}^{1} u^{(4)}(x) R_{y}^{(4)}(x) d x, \\
\left(u, R_{y} \in W_{2}^{4}[0,1]\right) .
\end{array}
$$

Through several integrations by parts for (21) we have

$$
\begin{aligned}
\left\langle u(x), R_{y}(x)\right\rangle_{W_{2}^{4}}= & \sum_{i=0}^{3} u^{(i)}(0)\left[R_{y}^{(i)}(0)-(-1)^{(3-i)} R_{y}^{(7-i)}(0)\right] \\
& +\sum_{i=0}^{3}(-1)^{(3-i)} u^{(i)}(1) R_{y}^{(7-i)}(1) \\
& +\int_{0}^{1} u(x) R_{y}^{(8)}(x) d x .
\end{aligned}
$$

Note that property of the reproducing kernel

$$
\left\langle u, R_{y}\right\rangle_{W_{2}^{4}}=u(y)
$$

$R_{y}$, is the solution of the following differential equation:

$$
R_{y}^{(8)}(x)=\delta(x-y)
$$

with the boundary conditions

$$
\begin{gathered}
R_{y}^{(3)}(0)-R_{y}^{(4)}(0)=0 \\
R_{y}^{\prime \prime}(0)+R_{y}^{(5)}(0)=0 \\
R_{y}^{(4)}(1)=0 \\
R_{y}^{(5)}(1)=0 \\
R_{y}^{(6)}(1)=0
\end{gathered}
$$

when $x \neq y$,

$$
R_{y}^{(8)}(x)=0
$$


therefore

$$
R_{y}(x)= \begin{cases}\sum_{i=1}^{8} c_{i}(y) x^{i-1}, & x \leq y \\ \sum_{i=1}^{8} d_{i}(y) x^{i-1}, & x>y\end{cases}
$$

Since

$$
R_{y}^{(8)}(x)=\delta(x-y)
$$

we have

$$
\begin{gathered}
\partial^{k} R_{y^{+}}(y)=\partial^{k} R_{y^{-}}(y), \quad k=0,1,2,3,4,5,6, \\
\partial^{7} R_{y^{+}}(y)-\partial^{7} R_{y^{-}}(y)=1 .
\end{gathered}
$$

Since $R_{y}(x) \in W_{2}^{4}[0,1]$, it follows that

$$
R_{y}(0)=0, \quad R_{y}^{\prime}(0)=0, \quad R_{y}(1)=0 .
$$

From (25)-(31), the unknown coefficients $c_{i}(y)$ ve $d_{i}(y)(i=$ $1,2, \ldots, 8)$ can be obtained.

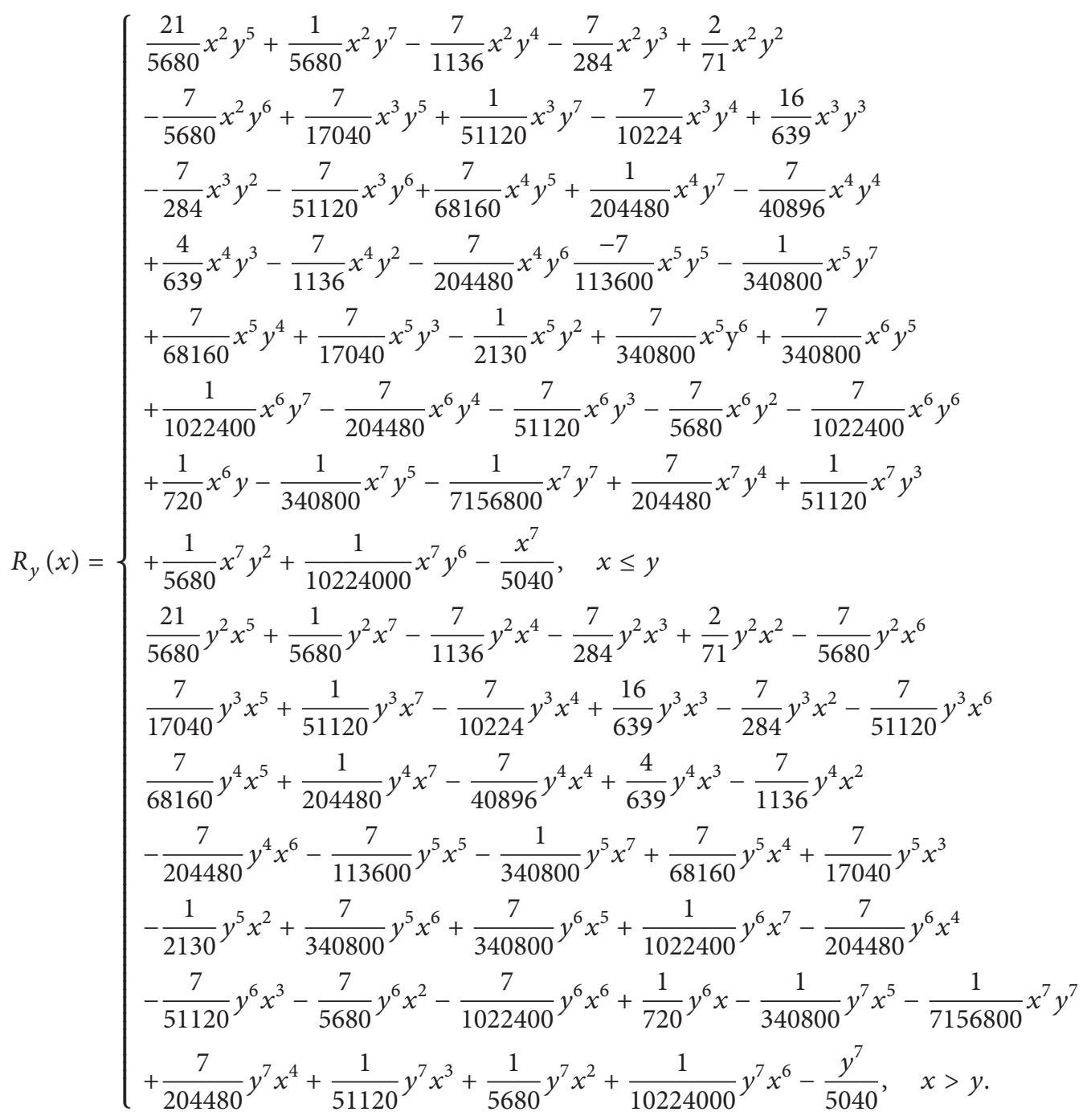

\section{Solution Representation in $W_{2}^{4}[0,1]$}

In this section, the solution of (34) is given in the reproducing kernel space $W_{2}^{4}[0,1]$.

On defining the linear operator $L: W_{2}^{4}[0,1] \rightarrow W_{2}^{2}[0,1]$ as

$$
(L u)(x)=u^{\prime \prime \prime}(x)
$$

$$
+\left[-2 \alpha \operatorname{Re}\left(x^{2}-1\right)+(4-H) \alpha^{2}\right] u^{\prime}(x)
$$$$
-4 \alpha x \operatorname{Re} u(x) \text {. }
$$

Model problem (6) changes the following problem:

$$
L u=f\left(x, u, u^{\prime}\right), \quad x \in[0,1],
$$




$$
u(0)=0, \quad u^{\prime}(0)=0, \quad u(1)=0,
$$

where

$$
\begin{aligned}
f\left(x, u, u^{\prime}\right)= & -2 \alpha \operatorname{Re} u(x) u^{\prime}(x)-4 \alpha \operatorname{Re}\left(x^{3}-x\right) \\
& +2(4-H) \alpha^{2} x, \\
& u(x)=F(x)+x^{2}-1 .
\end{aligned}
$$

Theorem 5. The operator L defined by (33) is a bounded linear operator.

Proof. We only need to prove $\|L u\|_{W_{2}^{2}}^{2} \leq M\|u\|_{W_{2}^{4}}^{2}$, where $M>$ 0 is a positive constant. By (15) and (16), we have

$$
\begin{aligned}
\|L u\|_{W_{2}^{2}}^{2}= & \langle L u, L u\rangle_{W_{2}^{2}} \\
= & {[(L u)(0)]^{2}+\left[(L u)^{\prime}(0)\right]^{2} } \\
& +\int_{0}^{1}\left[(L u)^{\prime \prime}(x)\right]^{2} d x .
\end{aligned}
$$

By (18), we have

$$
\begin{gathered}
u(x)=\left\langle u, R_{x}\right\rangle_{W_{2}^{4}}, \\
(L u)(x)=\left\langle u,\left(L R_{x}\right)\right\rangle_{W_{2}^{4}}, \\
(L u)^{\prime}(x)=\left\langle u,\left(L R_{x}\right)^{\prime}\right\rangle_{W_{2}^{4}},
\end{gathered}
$$

so

$$
|(L u)(x)| \leq\|u\|_{W_{2}^{4}}\left\|L R_{x}\right\|_{W_{2}^{4}}=M_{1}\|u\|_{W_{2}^{4}},
$$

(where $M_{1}>0$ is a positive constant),

$$
\left|(L u)^{\prime}(x)\right| \leq\|u\|_{W_{2}^{4}}\left\|\left(L R_{x}\right)^{\prime}\right\|_{W_{2}^{4}}=M_{2}\|u\|_{W_{2}^{4}},
$$

(where $M_{2}>0$ is a positive constant),

thus

$$
(L u)^{2}(0)+\left[(L u)^{\prime}(0)\right]^{2} \leq\left(M_{1}^{2}+M_{2}^{2}\right)\|u\|_{W_{2}^{4}}^{2} .
$$

Since

$$
(L u)^{\prime \prime}=\left\langle u,\left(L R_{x}\right)^{\prime \prime}\right\rangle_{W_{2}^{4}},
$$

then

$$
\left|(L u)^{\prime \prime}\right| \leq\|u\|_{W_{2}^{4}}\left\|\left(L R_{x}\right)^{\prime \prime}\right\|_{W_{2}^{4}}=M_{3}\|u\|_{W_{2}^{4}},
$$

(where $M_{3}>0$ is a positive constant),

so, we have

$$
\begin{gathered}
{\left[(L u)^{\prime \prime}\right]^{2} \leq M_{3}^{2}\|u\|_{W_{2}^{4}}^{2},} \\
\int_{0}^{1}\left[(L u)^{\prime \prime}(x)\right]^{2} d x \leq M_{3}^{2}\|u\|_{W_{2}^{4}}^{2},
\end{gathered}
$$

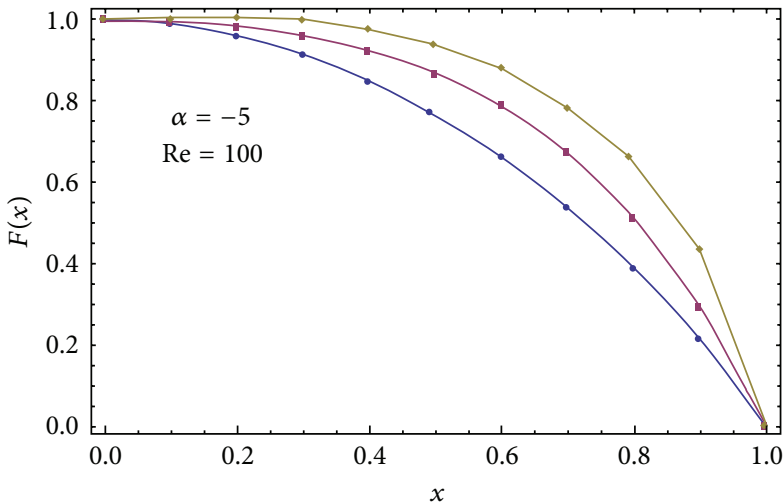

$$
\begin{aligned}
& \multimap H=1000 \\
& \because H=2000 \\
& \multimap H=3000
\end{aligned}
$$

FIGURE 2: A comparison between increasing Hartmann numbers for the velocity profile $\mathrm{Re}=100$.

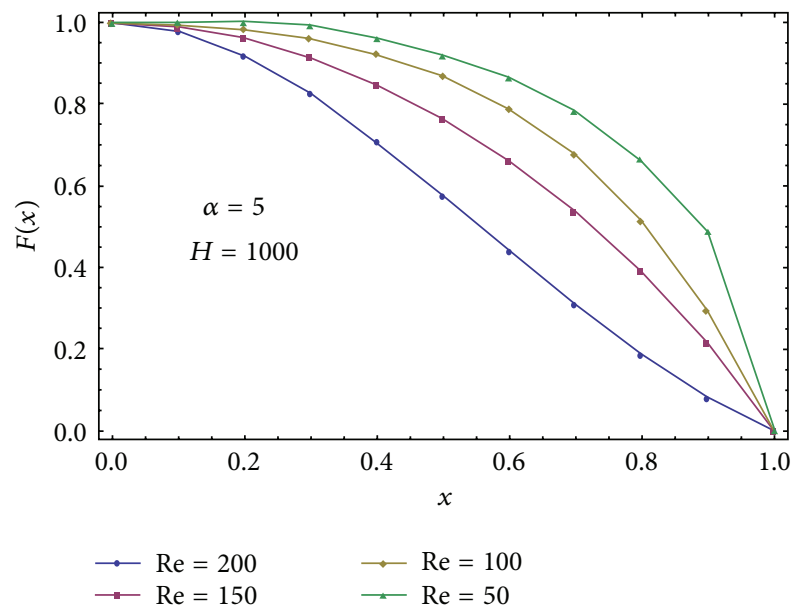

FIgURE 3: A comparison between the increasing values of Re for the velocity profile $H=1000$.

that is

$$
\begin{aligned}
\|L u\|_{W_{2}^{2}}^{2} & =[(L u)(0)]^{2}+\left[(L u)^{\prime}(0)\right]^{2}+\int_{0}^{1}\left[(L u)^{\prime \prime}(x)\right]^{2} d x \\
& \leq\left(M_{1}^{2}+M_{2}^{2}+M_{3}^{2}\right)\|u\|_{W_{2}^{4}}^{2}=M\|u\|_{W_{2}^{4}}^{2}
\end{aligned}
$$

where $M=\left(M_{1}^{2}+M_{2}^{2}+M_{3}^{2}\right)>0$ is a positive constant.

\section{The Structure of the Solution and the Main Results}

In (33) it is clear that $L: W_{2}^{4}[0,1] \rightarrow W_{2}^{2}[0,1]$ is a bounded linear operator. Put $\varphi_{i}=T_{x_{i}}$ and $\psi_{i}=L^{*} \varphi_{i}$, where $L^{*}$ is conjugate operator of $L$. The orthonormal system 


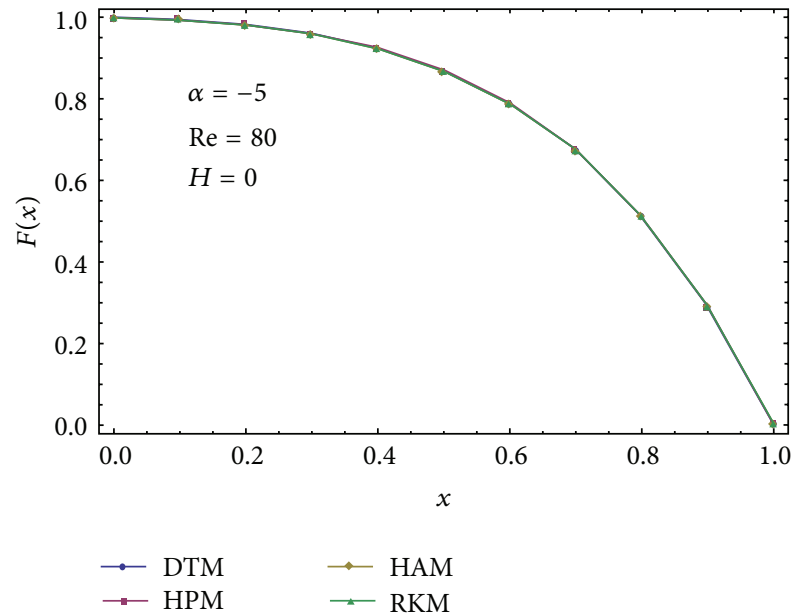

FIgUre 4: A comparison between the DTM, HPM, RKHSM, and HAM solutions for the velocity profile $\mathrm{Re}=80$ and $H=0$.

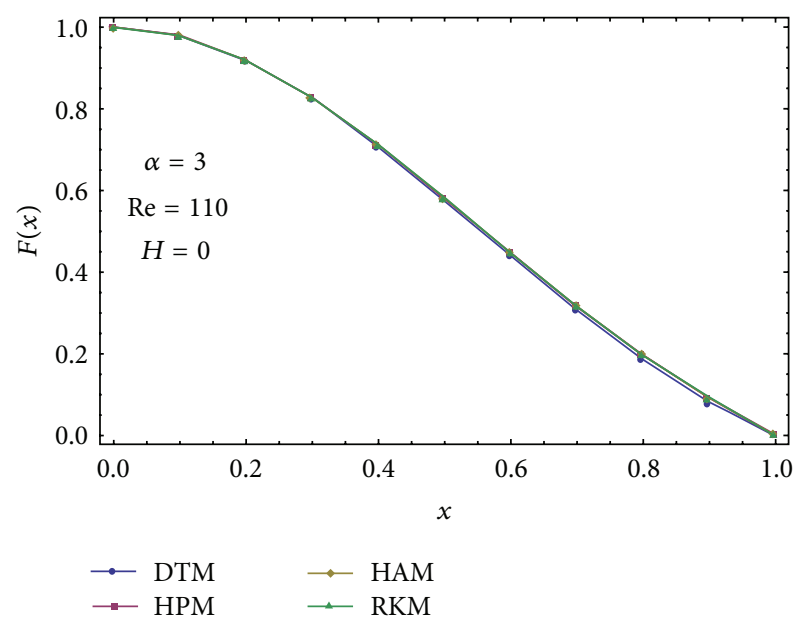

FIGURE 5: A comparison between the DTM, HPM, RKHSM, and HAM solutions for the velocity profile $\mathrm{Re}=110$ and $H=0$.

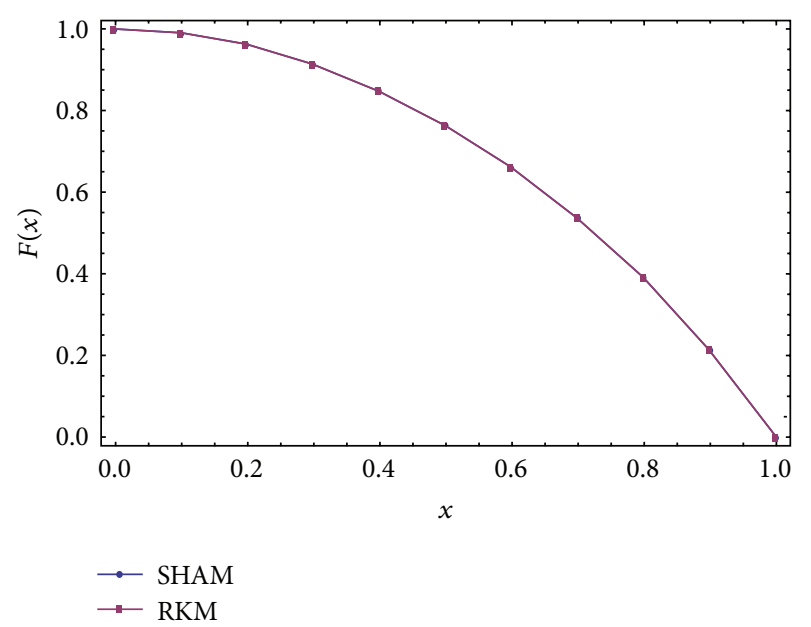

FIGURE 6: A comparison between the RKHSM and SHAM solutions for the velocity profile $\operatorname{Re}=50, \alpha=5$, and $H=0$.
$\left\{\bar{\Psi}_{i}\right\}_{i=1}^{\infty}$ of $W_{2}^{4}[0,1]$ can be derived from Gram-Schmidt orthogonalization process of $\left\{\psi_{i}\right\}_{i=1}^{\infty}$ as

$$
\bar{\psi}_{i}(x)=\sum_{k=1}^{i} \beta_{i k} \psi_{k}(x), \quad\left(\beta_{i i}>0, i=1,2, \ldots\right) .
$$

Theorem 6. For (33), if $\left\{x_{i}\right\}_{i=1}^{\infty}$ is dense on $[0,1]$ then $\left\{\psi_{i}\right\}_{i=1}^{\infty}$ is the complete system of $W_{2}^{4}[0,1]$ and $\psi_{i}(x)=\left.L_{y} R_{x}(y)\right|_{y=x_{i}}$.

Proof. We have

$$
\begin{aligned}
\psi_{i}(x) & =\left(L^{*} \varphi_{i}\right)(x)=\left\langle\left(L^{*} \varphi_{i}\right)(y), R_{x}(y)\right\rangle \\
& =\left\langle\left(\varphi_{i}\right)(y), L y R_{x}(y)\right\rangle=\left.L_{y} R_{x}(y)\right|_{y=x_{i}} .
\end{aligned}
$$

The subscript $y$ by the operator $L$ indicates that the operator $L$ applies to the function of $y$. Clearly, $\psi_{i}(x) \epsilon$ $W_{2}^{4}[0,1]$. For each fixed $u(x) \in W_{2}^{4}[0,1]$, let $\left\langle u(x), \psi_{i}(x)\right\rangle=$ $0, \quad(i=1,2, \ldots)$, which means that

$$
\left\langle u,\left(L^{*} \varphi_{i}\right)\right\rangle=\left\langle L u, \varphi_{i}\right\rangle=\left\langle L u, T_{x_{i}}\right\rangle=(L u)\left(x_{i}\right)=0 .
$$

Note that, $\left\{x_{i}\right\}_{i=1}^{\infty}$ is dense on $[0,1]$, hence, $(L u)(x)=0$. It follows that $u \equiv 0$ from the existence of $L^{-1}$. So the proof of Theorem 6 is complete.

Theorem 7. If $u(x)$ is the exact solution of (34), then

$$
u=\sum_{i=1}^{\infty} \sum_{k=1}^{i} \beta_{i k} f\left(x_{k}, u_{k}, u_{k}^{\prime}\right) \bar{\Psi}_{i},
$$

where $\left\{x_{i}\right\}_{i=1}^{\infty}$ is a dense set in $[0,1]$.

Proof. From (44) and uniqueness of solution of (34) we have

$$
\begin{aligned}
u & =\sum_{i=1}^{\infty}\left\langle u, \bar{\psi}_{i}\right\rangle_{W_{2}^{4}} \bar{\psi}_{i} \\
& =\sum_{i=1}^{\infty} \sum_{k=1}^{i} \beta_{i k}\left\langle u, L^{*} T_{x_{k}}\right\rangle_{W_{2}^{4}} \bar{\psi}_{i} \\
& =\sum_{i=1}^{\infty} \sum_{k=1}^{i} \beta_{i k}\left\langle L u, T_{x_{k}}\right\rangle_{W_{2}^{2}} \bar{\psi}_{i} \\
& =\sum_{i=1}^{\infty} \sum_{k=1}^{i} \beta_{i k}\left\langle f\left(x, u, u^{\prime}\right), T_{x_{k}}\right\rangle_{W_{2}^{2}} \bar{\psi}_{i} \\
& =\sum_{i=1}^{\infty} \sum_{k=1}^{i} \beta_{i k} f\left(x_{k}, u_{k}, u_{k}^{\prime}\right) \bar{\psi}_{i} .
\end{aligned}
$$

Now the approximate solution $u_{n}(x)$ can be obtained by truncating the $n$-term of the exact solution $u(x)$ :

$$
u_{n}=\sum_{i=1}^{n} \sum_{k=1}^{i} \beta_{i k} f\left(x_{k}, u_{k}, u_{k}^{\prime}\right) \bar{\psi}_{i}
$$


TABLE 1: The comparison between the numerical results and DTM, HPM, HAM, and RKHSM solutions for $\operatorname{Re}=110, \alpha=3$, and $H=0$.

\begin{tabular}{lccccc}
\hline$x$ & DTM [5] & HPM [5] & HAM [5] & RKHSM & Numerical [5] \\
\hline 0.0 & 1.000000000 & 1.0000000000 & 1.0000000000 & 1.0000000000 & 0.0792357171 \\
0.1 & 0.9789771156 & 0.9791761778 & 0.9792357062 & 0.91926585 & 0.9792357085 \\
0.2 & 0.9182598446 & 0.9190424983 & 0.9192658842 & 0.82653635 & 0.91926588998 \\
0.3 & 0.8243664466 & 0.8260939720 & 0.8265336102 & 0.7102315393 & 0.7102211890 \\
0.4 & 0.7065763476 & 0.7096036928 & 0.7102211838 & 0.5804817201 & 0.5804994634 \\
0.5 & 0.5751498602 & 0.5798357741 & 0.5804994700 & 0.4468796913 & 0.4469350697 \\
0.6 & 0.4397114086 & 0.4463900333 & 0.4469350941 & 0.3174013727 & 0.3174084270 \\
0.7 & 0.3081560927 & 0.3170877938 & 0.3174084545 & 0.1976321 & 0.1976410889 \\
0.8 & 0.1862239095 & 0.1975366451 & 0.1976410661 & 0.0912030082 & 0.0912304211 \\
0.9 & 0.0784362201 & 0.09124214542 & 0.09123022879 & $-0.052549207 \times 10^{-8}$ & 0.0 \\
1.0 & 0.0000000015 & 0.0000000007 & -0.00000047 &
\end{tabular}

TABLE 2: The numerical results for $\operatorname{Re}=50, H=1000$.

\begin{tabular}{|c|c|c|c|c|c|c|}
\hline$x$ & HAM [3] & RKHSM $(\alpha=5)$ & Error & HAM [3] & RKHSM $(\alpha=-5)$ & Error \\
\hline 0 & 1.000000000 & 1.0000000000 & 0.0 & 1.000000000 & 1.00000000 & 0.0 \\
\hline 0.05 & 0.997605126 & 0.997605447 & $3.203 \times 10^{-7}$ & 0.999197467 & 0.99919702 & $4.432 \times 10^{-7}$ \\
\hline 0.10 & 0.990427215 & 0.990432890 & $0.56744 \times 10^{-6}$ & 0.99675704 & 0.9967562 & $8.409 \times 10^{-7}$ \\
\hline 0.15 & 0.978485626 & 0.9784839628 & $0.16638 \times 10^{-6}$ & 0.992578975 & 0.992578 & $9.754 \times 10^{-7}$ \\
\hline 0.20 & 0.961810074 & 0.96179 & $0.20074 \times 10^{-5}$ & 0.98649281 & 0.98649340 & $5.900 \times 10^{-7}$ \\
\hline 0.25 & 0.940436864 & 0.9403939 & $0.42964 \times 10^{-5}$ & 0.978250927 & 0.9782510 & $7.24 \times 10^{-8}$ \\
\hline 0.30 & 0.91440365 & 0.9145 & $0.96349 \times 10^{-5}$ & 0.967519314 & 0.9675443 & $0.24985 \times 10^{-5}$ \\
\hline 0.35 & 0.883742856 & 0.8833 & $0.44285 \times 10^{-4}$ & 0.953865319 & 0.95382 & $0.45319 \times 10^{-5}$ \\
\hline 0.40 & 0.848473706 & 0.8484738539 & $1.473 \times 10^{-7}$ & 0.936742176 & 0.936821 & $0.78823 \times 10^{-5}$ \\
\hline 0.45 & 0.808592961 & 0.808592834 & $1.279 \times 10^{-7}$ & 0.915470063 & 0.915531 & $0.60936 \times 10^{-5}$ \\
\hline 0.50 & 0.764064241 & 0.7640637445 & $4.967 \times 10^{-7}$ & 0.889213540 & 0.889241 & $0.27459 \times 10^{-5}$ \\
\hline 0.55 & 0.714805913 & 0.7148062 & $2.867 \times 10^{-7}$ & 0.856955292 & 0.8565 & $0.45529 \times 10^{-4}$ \\
\hline 0.60 & 0.660677266 & 0.660670 & $0.72666 \times 10^{-6}$ & 0.817466464 & 0.817199 & $0.26746 \times 10^{-4}$ \\
\hline 0.65 & 0.601462467 & 0.6014683135 & $0.58461 \times 10^{-6}$ & 0.769274094 & 0.770 & $0.7259 \times 10^{-4}$ \\
\hline 0.70 & 0.536852087 & 0.53685274 & $6.525 \times 10^{-7}$ & 0.710627559 & 0.710014 & $0.61355 \times 10^{-4}$ \\
\hline 0.75 & 0.466421078 & 0.4664202 & $8.783 \times 10^{-7}$ & 0.639465773 & 0.63946970 & $0.39331 \times 10^{-6}$ \\
\hline 0.80 & 0.389601905 & 0.389602099 & $1.934 \times 10^{-7}$ & 0.553390063 & 0.55336107 & $0.28992 \times 10^{-5}$ \\
\hline 0.85 & 0.305651801 & 0.305645 & $0.68011 \times 10^{-6}$ & 0.449648596 & 0.44963621 & $0.12386 \times 10^{-5}$ \\
\hline 0.90 & 0.213611172 & 0.2136120 & $8.277 \times 10^{-7}$ & 0.325142373 & 0.32516167 & $0.19298 \times 10^{-5}$ \\
\hline 0.95 & 0.112250324 & 0.112249347 & $9.775 \times 10^{-7}$ & 0.176465831 & 0.17656197 & $0.9614 \times 10^{-5}$ \\
\hline 1.00 & 0.000000000 & $8.3437 \times 10^{-8}$ & $8.3437 \times 10^{-8}$ & 0.000000000 & $3.614 \times 10^{-7}$ & $3.614 \times 10^{-7}$ \\
\hline
\end{tabular}

TABLE 3: The comparison between the numerical results and DTM, HPM, HAM, and RKHSM solutions for Re $=80, \alpha=-5$, and $H=0$.

\begin{tabular}{lccccc}
\hline$x$ & DTM [5] & HPM [5] & HAM [5] & RKHSM & Numerical \\
\hline 0 & 1.000000000 & 1.0000000000 & 1.0000000000 & 1.0000000000 & 1.0000000000 \\
0.10 & 0.9959603887 & 0.9960671874 & 0.9959606242 & 0.99595999 & 0.9959606278 \\
0.20 & 0.9832745481 & 0.9836959424 & 0.9832755258 & 0.983275 & 0.9832755381 \\
0.30 & 0.9601775551 & 0.9610758773 & 0.9601798911 & 0.96017 & 0.96017991139 \\
0.40 & 0.9235170706 & 0.9249245156 & 0.9235215737 & 0.923519 & 0.9235215894 \\
0.50 & 0.8684511349 & 0.8701997697 & 0.8684588997 & 0.86845826 & 0.86845887772 \\
0.60 & 0.7880785402 & 0.7898325937 & 0.7880910186 & 0.78809 & 0.78809092032 \\
0.70 & 0.6731248448 & 0.6745334968 & 0.6731437690 & 0.67314 & 0.6731436346 \\
0.80 & 0.5119644061 & 0.5128373095 & 0.5119909939 & 0.5119873503 & 0.5119910891 \\
0.90 & 0.2915280122 & 0.2918936991 & 0.2915580178 & 0.2915582665 & 0.29155874261 \\
1.00 & 0.0000000000 & 0.0000000001 & -0.000001149 & $2.851385 \times 10^{-9}$ & 0.0 \\
\hline
\end{tabular}


Lemma 8. If $u \in W_{2}^{4}[0,1]$, then there exists $M_{1}>0$, such that

$$
\|u\|_{C^{2}[0,1]} \leq M_{1}\|u\|_{W_{2}^{4}}
$$

where $\|u\|_{C^{2}[0,1]}=\max _{x \in[0,1]}|u(x)|+\max _{x \in[0,1]}\left|u^{\prime}(x)\right|+$ $\max _{x \in[0,1]}\left|u^{\prime \prime}(x)\right|$.

Lemma 9. If $\left\|u_{n}-u\right\|_{W_{2}^{4}} \rightarrow 0, x_{n} \rightarrow x,(n \rightarrow \infty)$ and $f\left(x, u, u^{\prime}\right)$ is continuous for $\left.x \in 0,1\right]$, then

$$
\begin{aligned}
& f\left(x_{n}, u_{n-1}\left(x_{n}\right), u_{n-1}^{\prime}\left(x_{n}\right)\right) \\
& \quad \longrightarrow f\left(x, u(x), u^{\prime}(x)\right) \text { as } n \longrightarrow \infty .
\end{aligned}
$$

Proof. Since $\left\|u_{n}-u\right\|_{W_{2}^{4}} \rightarrow 0(n \rightarrow \infty)$, by Lemma 8 , we know that $u_{n}$ is convergent uniformly to $u(x)$, therefore, the proof is complete.

Remark 10. (i) If (34) is linear, that is, $f(x, u)=f(x)$, then the analytical solution of (34) can be obtained directly by (47).

(ii) If (34) is nonlinear; that is, $f$ depends on $u$ and $u^{\prime}$ then the solution of (34) can be obtained by the following iterative method.

We construct an iterative sequence $u_{n}(x)$, putting any fixed $u_{0}(x) \in W_{2}^{4}[0,1]$,

$$
u_{n}(x)=\sum_{i=1}^{n} A_{i} \bar{\psi}_{i}(x)
$$

where

$$
\begin{aligned}
A_{1}= & \beta_{11} f\left(x_{1}, u_{0}\left(x_{1}\right), u_{0}^{\prime}\left(x_{1}\right)\right), \\
A_{2}= & \sum_{k=1}^{2} \beta_{2 k} f\left(x_{k}, u_{k-1}\left(x_{k}\right), u_{k-1}^{\prime}\left(x_{k}\right)\right), \\
& \vdots \\
A_{n}= & \sum_{k=1}^{n} \beta_{n k} f\left(x_{k}, u_{k-1}\left(x_{k}\right), u_{k-1}^{\prime}\left(x_{k}\right)\right) .
\end{aligned}
$$

Next we will prove that $u_{n}$ given by the iterative formula (52) converges to the exact solution (47).

Theorem 11. Suppose that the following conditions are satisfied: (i) $\left\|u_{n}\right\|_{W_{2}^{4}}$ is bounded; (ii) $\left\{x_{i}\right\}_{i=1}^{\infty}$ is a dense in $[0,1]$; (iii) $f\left(x, u, u^{\prime}\right) \in W_{2}^{2}[0,1]$ for any $u \in W_{2}^{4}[0,1]$. Then $u_{n}$ in iterative formula (52) converges to the exact solution of (47) in $W_{2}^{4}[0,1]$ and

$$
u=\sum_{i=1}^{\infty} A_{i} \bar{\psi}_{i}
$$

where $A_{i}$ is given by (53).
Proof. (i) First, we will prove the convergence of $u_{n}(x)$. By (52), we have

$$
u_{n+1}(x)=u_{n}(x)+A_{n+1} \bar{\psi}_{n+1}(x) .
$$

From the orthogonality of $\left\{\bar{\Psi}_{i}(x)\right\}_{i=1}^{\infty}$, it follows that

$$
\begin{aligned}
\left\|u_{n+1}\right\|_{W_{2}^{4}}^{2} & =\left\|u_{n}\right\|_{W_{2}^{4}}^{2}+\left(A_{n+1}\right)^{2} \\
& =\left\|u_{n-1}\right\|_{W_{2}^{4}}^{2}+\left(A_{n}\right)^{2}+\left(A_{n+1}\right)^{2} \\
& =\cdots=\sum_{i=1}^{n+1}\left(A_{i}\right)^{2} .
\end{aligned}
$$

From boundedness of $\left\|u_{n}\right\|_{W_{2}^{4}}$, we have

$$
\sum_{i=1}^{\infty}\left(A_{i}\right)^{2}<\infty
$$

that is,

$$
\left\{A_{i}\right\} \in l^{2} \quad(i=1,2, \ldots) .
$$

Let $m>n$, in view of $\left(u_{m}-u_{m-1}\right) \perp\left(u_{m-1}-u_{m-2}\right) \perp \cdots \perp$ $\left(u_{n+1}-u_{n}\right)$, it follows that

$$
\begin{aligned}
\| u_{m}- & u_{n} \|_{W_{2}^{4}}^{2} \\
& =\left\|u_{m}-u_{m-1}+u_{m-1}-\mathrm{u}_{m-2}+\cdots+u_{n+1}-u_{n}\right\|_{W_{2}^{4}}^{2} \\
& \leq\left\|u_{m}-u_{m-1}\right\|_{W_{2}^{4}}^{2}+\cdots+\left\|u_{n+1}-u_{n}\right\|_{W_{2}^{4}}^{2} \\
& =\sum_{i=n+1}^{m}\left(A_{i}\right)^{2} \longrightarrow 0 \quad(m, n \longrightarrow \infty) .
\end{aligned}
$$

Considering the completeness of $W_{2}^{4}[0,1]$, there exists $u(x) \epsilon$ $W_{2}^{4}[0,1]$, such that

$$
u_{n}(x) \stackrel{\|\cdot\|_{W_{2}^{4}}}{\longrightarrow} u(x), \quad \text { as } n \longrightarrow \infty .
$$

(ii) Second, we will prove that $u(x)$ is the solution of (34).

By Lemma 8 and Theorem 11 (i), we know that $u_{n}$ converges uniformly to $u$. It follows that, on taking limits in (52),

$$
u=\sum_{i=1}^{\infty} A_{i} \bar{\psi}_{i}
$$

Since

$$
\begin{aligned}
(L u)\left(x_{j}\right) & =\sum_{i=1}^{\infty} A_{i}\left\langle L \bar{\Psi}_{i}(x), \varphi_{j}(x)\right\rangle_{W_{2}^{2}} \\
& =\sum_{i=1}^{\infty} A_{i}\left\langle\bar{\Psi}_{i}(x), L^{*} \varphi_{j}(x)\right\rangle_{W_{2}^{4}} \\
& =\sum_{i=1}^{\infty} A_{i}\left\langle\bar{\Psi}_{i}(x), \bar{\Psi}_{j}(x)\right\rangle_{W_{2}^{4}},
\end{aligned}
$$


TABLE 4: The errors of DTM, HPM, HAM, and RKHSM for $F(x)$ results when $\operatorname{Re}=110, \alpha=3$, and $H=0$.

\begin{tabular}{ccccc}
\hline$x$ & DTM [5] & HPM [5] & HAM [5] & RKHSM \\
\hline 0.0 & 0.0 & 0.0 & 0.0 & 0.0 \\
0.1 & 0.0002 & 0.000059 & 0.0000000023 & $8.6 \times 10^{-9}$ \\
0.2 & 0.0010 & 0.00022 & 0.0000000056 & $3.98 \times 10^{-8}$ \\
0.3 & 0.0021 & 0.00043 & 0.000000008 & 0.0000027318 \\
0.4 & 0.0036 & 0.00061 & 0.0000000052 & 0.0000103503 \\
0.5 & 0.0053 & 0.00066 & 0.0000000066 & 0.0000177433 \\
0.6 & 0.0072 & 0.00054 & 0.000000024 & 0.0000553784 \\
0.7 & 0.0092 & 0.00032 & 0.000000027 & 0.0000070543 \\
0.8 & 0.0114 & 0.000104 & 0.000000022 & 0.0000089889 \\
0.9 & 0.0127 & 0.000011 & 0.00000019 & 0.0000274129 \\
1.0 & 0.0000 & 0.000000 & 0.0000004 & $8.052549207 \times 10^{-8}$ \\
\hline
\end{tabular}

TABle 5: The errors of DTM, HPM, HAM, and RKHSM for $F(x)$ results when for $\operatorname{Re}=80, \alpha=-5$, and $H=0$.

\begin{tabular}{lcccc}
\hline$x$ & DTM [5] & HPM [5] & HAM [5] & RKHSM \\
\hline 0.0 & 0.0 & 0.0 & 0.0 & 0.0 \\
0.1 & 0.00000023 & 0.000106 & 0.000000003 & $6.378 \times 10^{-7}$ \\
0.2 & 0.00000099 & 0.00042 & 0.000000012 & $5.381 \times 10^{-7}$ \\
0.3 & 0.0000023 & 0.00089 & 0.00000002 & $0.9114 \times 10^{-6}$ \\
0.4 & 0.0000045 & 0.0014 & 0.000000015 & $2.5894 \times 10^{-6}$ \\
0.5 & 0.0000077 & 0.0017 & 0.000000021 & $6.177 \times 10^{-7}$ \\
0.6 & 0.000012 & 0.0017 & 0.000000098 & $9.203 \times 10^{-7}$ \\
0.7 & 0.000018 & 0.0013 & 0.00000013 & $3.6346 \times 10^{-6}$ \\
0.8 & 0.000026 & 0.0008 & 0.000000095 & $7.388 \times 10^{-6}$ \\
0.9 & 0.000030 & 0.00033 & 0.00000072 & $4.761 \times 10^{-7}$ \\
1.0 & 0.0000 & 0.0000000001 & 0.0000011 & $2.8513856 \times 10^{-9}$ \\
\hline
\end{tabular}

it follows that

$$
\begin{aligned}
\sum_{j=1}^{n} \beta_{n j}(L u)\left(x_{j}\right) & =\sum_{i=1}^{\infty} A_{i}\left\langle\bar{\Psi}_{i}(x), \sum_{j=1}^{n} \beta_{n j} \bar{\Psi}_{j}(x)\right\rangle_{W_{2}^{4}} \\
& =\sum_{i=1}^{\infty} A_{i}\left\langle\bar{\Psi}_{i}(x), \bar{\Psi}_{n}(x)\right\rangle_{W_{2}^{4}}=A_{n} .
\end{aligned}
$$

If $n=1$, then

$$
(L u)\left(x_{1}\right)=f\left(x_{1}, u_{0}\left(x_{1}\right), u_{0}^{\prime}\left(x_{1}\right)\right) .
$$

If $n=2$, then

$$
\begin{aligned}
\beta_{21}(L u) & \left(x_{1}\right)+\beta_{22}(L u)\left(x_{2}\right) \\
= & \beta_{21} f\left(x_{1}, u_{0}\left(x_{1}\right), u_{0}^{\prime}\left(x_{1}\right)\right) \\
& +\beta_{22} f\left(x_{2}, u_{1}\left(x_{2}\right), u_{1}^{\prime}\left(x_{2}\right)\right) .
\end{aligned}
$$

From (64) and (65), it is clear that

$$
(L u)\left(x_{2}\right)=f\left(x_{2}, u_{1}\left(x_{2}\right), u_{1}^{\prime}\left(x_{2}\right)\right) \text {. }
$$

Furthermore, it is easy to see by induction that

$$
(L u)\left(x_{j}\right)=f\left(x_{j}, u_{j-1}\left(x_{j}\right), u_{j-1}^{\prime}\left(x_{j}\right)\right) \text {. }
$$

Notice that $\left\{x_{i}\right\}_{i=1}^{\infty}$ is dense on interval $[0,1]$, for any $y \in[0,1]$, there exists subsequence $\left\{x_{n_{j}}\right\}$, such that $x_{n_{j}} \rightarrow y$, as $j \rightarrow$ $\infty$. Hence, by the convergence of $u_{n}(x)$ and Lemma 9, we have

$$
(L u)(y)=f\left(y, u(y), u^{\prime}(y)\right)
$$

that is, $u(x)$ is the solution of (34) and

$$
u=\sum_{i=1}^{\infty} A_{i} \bar{\psi}_{i}
$$

where $A_{i}$ is given by (53).

Corollary 12. Assume that the conditions of Theorem 11 hold; then $u_{n}$ in (52) satisfies $\left\|u_{n}-u\right\|_{C^{2}[0,1]} \rightarrow 0, n \rightarrow \infty$, where $u$ is the solution of (34).

Theorem 13. Assume that $u$ is the solution of (34) and $r_{n}$ is the error between the approximate solution $u_{n}$ and the exact solution $u$. Then the error sequence $r_{n}$ is monotone decreasing in the sense of $\|\cdot\|_{W_{2}^{4}}$ and $\left\|r_{n}(x)\right\|_{W_{2}^{4}} \rightarrow 0$.

Proof. From (47) and (49), it follows that

$$
\begin{aligned}
\left\|r_{n}\right\|_{W_{2}^{4}} & =\left\|\sum_{i=n+1}^{\infty} \sum_{k=1}^{i} \beta_{i k} f\left(x_{k}, u_{k}, u_{k}^{\prime}\right) \bar{\psi}_{i}(x)\right\|_{W_{2}^{4}} \\
& =\sum_{i=n+1}^{\infty}\left(\sum_{k=1}^{i} \beta_{i k} f\left(x_{k}, u_{k}, u_{k}^{\prime}\right)\right)^{2} .
\end{aligned}
$$

Equation (70) shows that the error $r_{n}$ is decreasing in the sense of $\|\cdot\|_{W_{2}^{4}}$.

\section{Numerical Results}

All computations are performed by Maple 15. Results obtained by the method are compared with the homotopy analysis method [3], three analytical methods [5], homotopy perturbation method [6], and a new spectral-homotopy analysis method [8]. The RKHSM does not require discretization of the variables, that is, time and space; it is not effected by computation round off errors and one is not faced with necessity of large computer memory and time. The accuracy of the RKHSM for the MHD Jeffery-Hamel flows problem is controllable and absolute errors are small with present choice of $x$ (see Tables 1-5). The numerical results that we obtained justify the advantage of this methodology.

5.1. Result and Discussion. In this study the purpose is to apply the RKHSM to obtain an approximate solution of the Jeffery-Hamel problem. The obtained results of RKHSM solution and numerical ones are shown in the tables and figures. In Table 2 a comparison of the HAM and RKHSM is shown. Tables 1 and 3 show the comparison between the numerical results and DTM, HPM, HAM, and RKHSM solutions. Tables 4 and 5 indicate the errors of DTM, HPM, HAM, and RKHSM for $F(x)$ results. Our results further show 


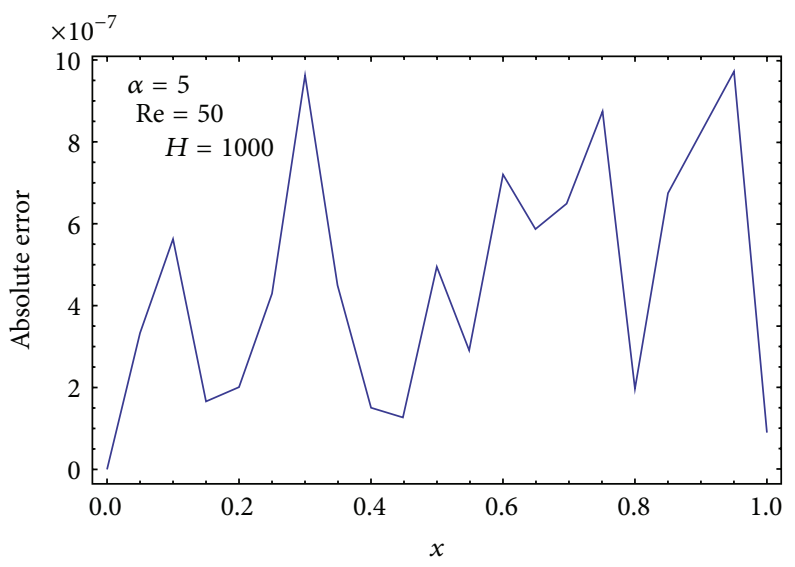

FIGURE 7: Absolute error for $\operatorname{Re}=50$ and $H=1000$.

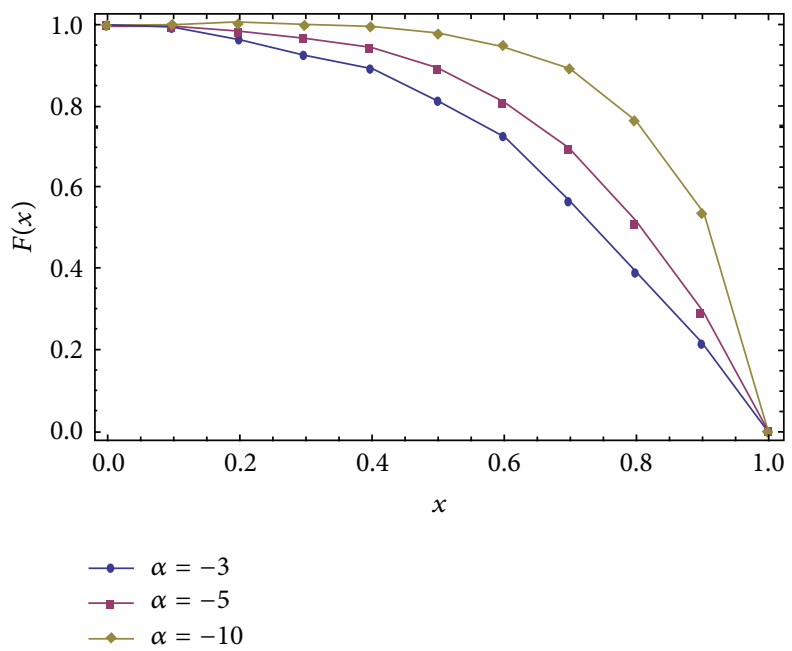

FIGURE 8: A comparison between different values of $\alpha$ for velocity in convergent channel for $\operatorname{Re}=50$ and $H=1000$.

that the fluid velocity increases with increasing Hartman numbers. Numerical simulations show that for fixed Hartmann numbers, the fluid velocity increases with Reynolds numbers in the case of convergent channels but decreases with $\mathrm{Re}$ in the case of divergent channels. Figure 2 indicates that increasing the Hartmann number leads to higher velocity which has a great effect on the performance of the system. In Figure 3 we give a comparison between the RKHSM and the HAM solutions for several Re numbers at $H=1000$. In Figure 4 we can see a comparison between the DTM, HPM, RKHSM and HAM solutions for the velocity profile $\mathrm{Re}=$ 80 and $H=0$. There is a comparison between the DTM, HPM, RKHSM, and HAM solutions for the velocity profile $\mathrm{Re}=110$ and $H=0$ in Figure 5. In Figure 6 we compare RKHSM and SHAM solutions. We can see absolute error for $\operatorname{Re}=50$ and $H=1000$ in Figure 7. The comparison of numerical results and RKHSM solution for velocity in convergent channel for $\operatorname{Re}=50$ and $H=1000$ is given with Figure 8. The solutions show that the results of the present method are in excellent agreement with those of the numerical ones. Moreover, RKHSM has been used to investigate the effects of the parameters of the problem.

\section{Conclusion}

In this paper, we introduce an algorithm for solving the MHD Jeffery-Hamel flows problem with boundary conditions by using the RKHSM. The approximate solution obtained by the present method is uniformly convergent. Clearly, the series solution methodology can be applied to much more complicated nonlinear differential equations and boundary value problems. However, if the problem becomes nonlinear, then the RKHSM does not require discretization or perturbation and it does not make closure approximation. Results show that the present method is an accurate and reliable analytical method for MHD Jeffery-Hamel flows problem with boundary conditions.

\section{Conflict of Interests}

The authors declare that they do not have any competing or conflict of interests.

\section{Acknowledgment}

The first and second authors acknowledge that this researchs supported by Firat University Scientific Research Projects 
Unit, Turkey is under the Research University Grant Scheme FF.12.09.

\section{References}

[1] G. B. Jeffery, "The two-dimensional steady motion of a viscous fluid," Philosophical Magazine, vol. 29, no. 172, pp. 455-465, 1915.

[2] G. Hamel, S. Bewgungen, and Z. Flussigkeiten, "Jahresbericht der Deutschen," Mathematiker-Vereinigung, vol. 25, pp. 34-60, 1916.

[3] S. M. Moghimi, G. Domairry, S. Soleimani, E. Ghasemi, and H. Bararnia, "Application of homotopy analysis method to solve MHD Jeffery-Hamel flows in non-parallel walls," Advances in Engineering Software, vol. 42, no. 3, pp. 108-113, 2011.

[4] M. Esmaeilpour and D. D. Ganji, "Solution of the JefferyHamel flow problem by optimal homotopy asymptotic method," Computers \& Mathematics with Applications, vol. 59, no. 11, pp. 3405-3411, 2010.

[5] A. A. Joneidi, G. Domairry, and M. Babaelahi, "Three analytical methods applied to Jeffery-Hamel flow," Communications in Nonlinear Science and Numerical Simulation, vol. 15, no. 11, pp. 3423-3434, 2010.

[6] S. M. Moghimi, D. D. Ganji, H. Bararnia, M. Hosseini, and M. Jalaal, "Homotopy perturbation method for nonlinear MHD Jeffery-Hamel problem," Computers \& Mathematics with Applications, vol. 61, no. 8, pp. 2213-2216, 2011.

[7] Q. Esmaili, A. Ramiar, E. Alizadeh, and D. D. Ganji, "An approximation of the analytical solution of the Jeffery-Hamel flow by decomposition method," Physics Letters, vol. 372, no. 19, pp. 3434-3439, 2008.

[8] S. S. Motsa, P. Sibanda, F. G. Awad, and S. Shateyi, "A new spectral-homotopy analysis method for the MHD JefferyHamel problem," Computers \& Fluids, vol. 39, no. 7, pp. 12191225, 2010.

[9] S. Goldstein, Modem Developments in Fluid Dynamics, vol. 1, Clarendon Press, Oxford, UK, 1938.

[10] W. I. Axford, "The magnetohydrodynamic Jeffrey-Hamel problem for a weakly conducting fluid," The Quarterly Journal of Mechanics and Applied Mathematics, vol. 14, pp. 335-351, 1961.

[11] S. Abbasbandy and E. Shivanian, "Exact analytical solution of the MHD Jeffery-Hamel fow problem," Mecannica, vol. 47, no. 6, pp. 1379-1389, 2012.

[12] O. D. Makinde, "Effect of arbitrary magnetic Reynolds number on MHD flows in convergent-divergent channels," International Journal of Numerical Methods for Heat \& Fluid Flow, vol. 18, no. 5-6, pp. 697-707, 2008.

[13] O. D. Makinde and P. Y. Mhone, "Hermite-Padé approximation approach to MHD Jeffery-Hamel flows," Applied Mathematics and Computation, vol. 181, no. 2, pp. 966-972, 2006.

[14] N. Aronszajn, “Theory of reproducing kernels," Transactions of the American Mathematical Society, vol. 68, pp. 337-404, 1950.

[15] M. Cui and Y. Lin, Nonlinear Numerical Analysis in the Reproducing Kernel Space, Nova Science Publishers, New York, NY, USA, 2009.

[16] F. Geng and M. Cui, "Solving a nonlinear system of second order boundary value problems," Journal of Mathematical Analysis and Applications, vol. 327, no. 2, pp. 1167-1181, 2007.

[17] F. Geng, "A new reproducing kernel Hilbert space method for solving nonlinear fourth-order boundary value problems," Applied Mathematics and Computation, vol. 213, no. 1, pp. 163169, 2009.
[18] F. Geng and M. Cui, "New method based on the HPM and RKHSM for solving forced Duffing equations with integral boundary conditions," Journal of Computational and Applied Mathematics, vol. 233, no. 2, pp. 165-172, 2009.

[19] F. Geng, M. Cui, and B. Zhang, "Method for solving nonlinear initial value problems by combining homotopy perturbation and reproducing kernel Hilbert space methods," Nonlinear Analysis, vol. 11, no. 2, pp. 637-644, 2010.

[20] F. Geng and M. Cui, "Homotopy perturbation-reproducing kernel method for nonlinear systems of second order boundary value problems," Journal of Computational and Applied Mathematics, vol. 235, no. 8, pp. 2405-2411, 2011.

[21] F. Geng and M. Cui, "A novel method for nonlinear two-point boundary value problems: combination of ADM and RKM," Applied Mathematics and Computation, vol. 217, no. 9, pp. 46764681, 2011.

[22] M. Mohammadi and R. Mokhtari, "Solving the generalized regularized long wave equation on the basis of a reproducing kernel space," Journal of Computational and Applied Mathematics, vol. 235, no. 14, pp. 4003-4014, 2011.

[23] W. Jiang and Y. Lin, "Representation of exact solution for the time-fractional telegraph equation in the reproducing kernel space," Communications in Nonlinear Science and Numerical Simulation, vol. 16, no. 9, pp. 3639-3645, 2011.

[24] Y. Wang, L. Su, X. Cao, and X. Li, "Using reproducing kernel for solving a class of singularly perturbed problems," Computers \& Mathematics with Applications, vol. 61, no. 2, pp. 421-430, 2011.

[25] B. Y. Wu and X. Y. Li, "A new algorithm for a class of linear nonlocal boundary value problems based on the reproducing kernel method," Applied Mathematics Letters, vol. 24, no. 2, pp. 156-159, 2011.

[26] H. Yao and Y. Lin, "New algorithm for solving a nonlinear hyperbolic telegraph equation with an integral condition," International Journal for Numerical Methods in Biomedical Engineering, vol. 27, no. 10, pp. 1558-1568, 2011.

[27] F. Geng and M. Cui, "A reproducing kernel method for solving nonlocal fractional boundary value problems," Applied Mathematics Letters, vol. 25, no. 5, pp. 818-823, 2012.

[28] M. Inc and A. Akgül, "The reproducing kernel hilbert space method for solving troesch's problem," Journal of the Association of Arab Universities For Basic and Applied Sciences, 2013.

[29] M. Inc, A. Akgül, and F. Geng, "Reproducing kernel hilbert space method for solving bratu's problem," Bulletin of the Malaysian Mathematical Sciences Society. In press.

[30] M. Inc, A. Akgül, and A. Kiliçman, "Explicit solution of telegraph equation based on reproducing kernel method," Journal of Function Spaces and Applications, vol. 2012, Article ID 984682, 23 pages, 2012.

[31] M. Inc, A. Akgül, and A. Kiliçman, "A novel method for solving $\mathrm{KdV}$ equation based on reproducing Kernel Hilbert space method," Abstract and Applied Analysis, vol. 2013, Article ID 578942, 11 pages, 2013. 


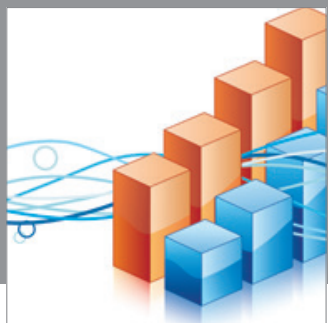

Advances in

Operations Research

mansans

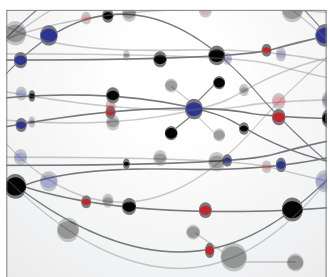

The Scientific World Journal
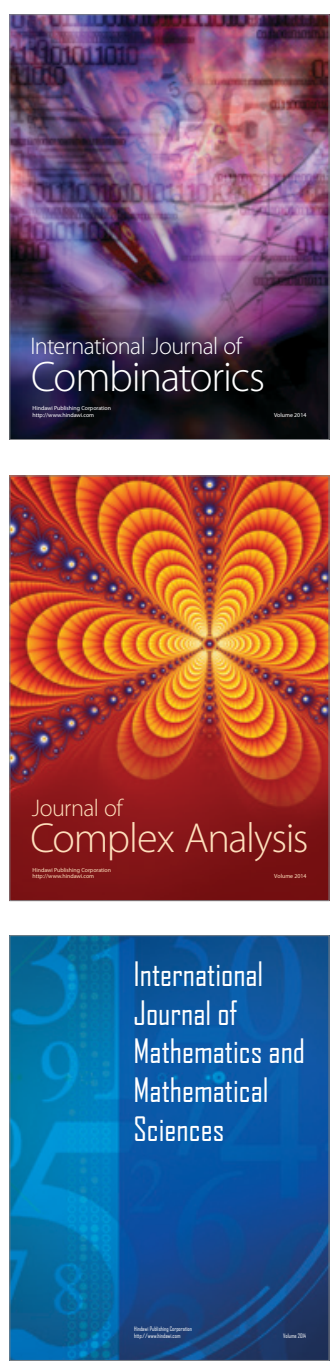
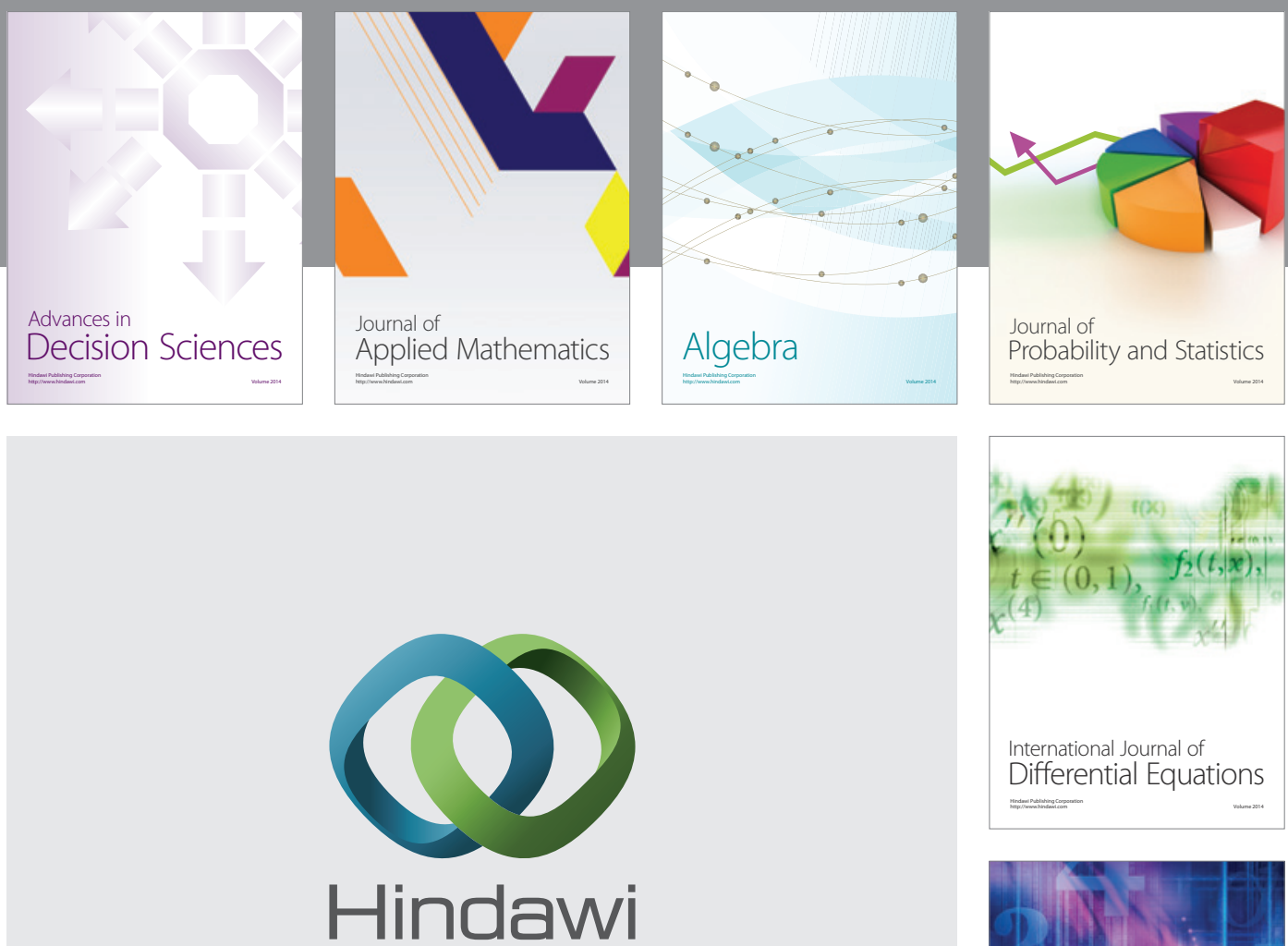

Submit your manuscripts at http://www.hindawi.com
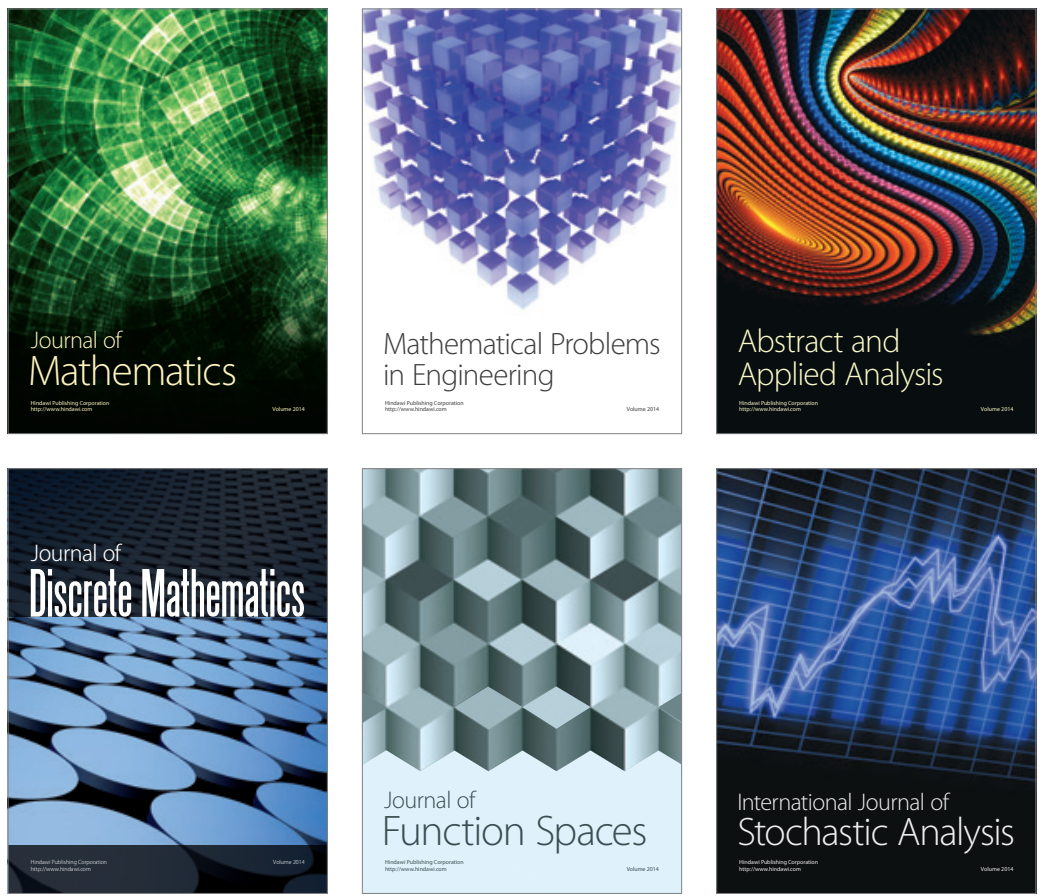

Journal of

Function Spaces

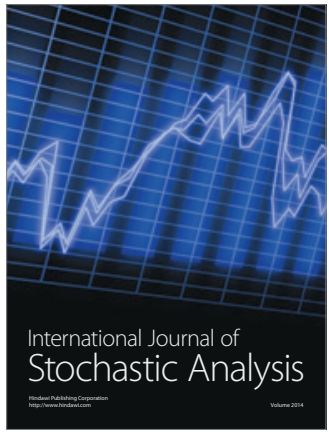

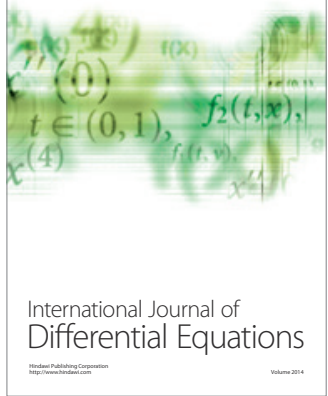
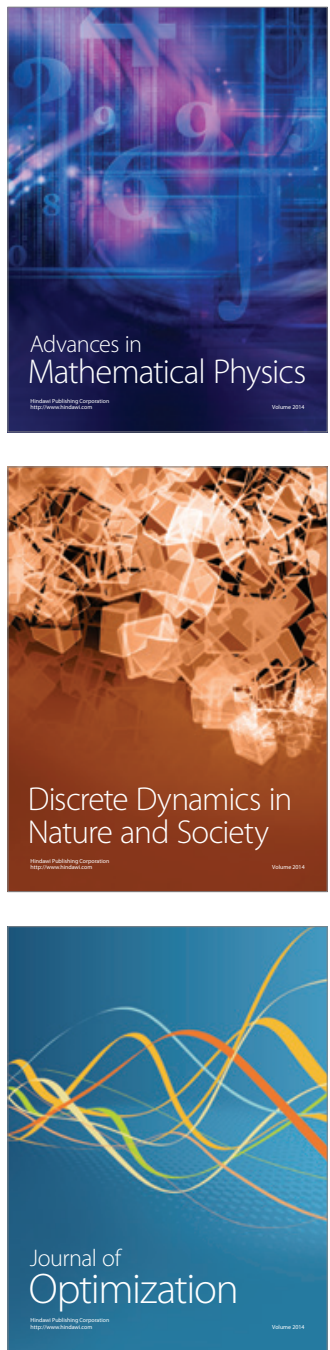